\title{
Die Enteignung von Grundbesitz zur Landreform in Südafrika im Spiegel des Verfassungsrechts und des einfachen Rechts
}

\author{
Von Konstantin Krukowski, Berlin
}

\section{A. Einleitung: Landreform im Spannungsfeld von Eigentumsschutz und Wiedergutmachung von Apartheidsunrecht}

Ende September 2005 sind zur Durchsetzung der Landreform in der Republik Südafrika von der Land Claims Commission für die Provinzen Gauteng und Nordwest die ersten Enteignungsverfahren gegen weiße Großgrundbesitzer eingeleitet worden. ${ }^{1}$ Dies fand in der internationalen Presse kurz Widerhall und ist deshalb bemerkenswert, weil Südafrika in den mittlerweile mehr als zehn Jahren als funktionierender freiheitlich-demokratischer Rechtsstaat $^{2}$ auf dem Gebiet der Landreform und der allmählichen Übertragung von Grund und Boden an die zur Zeit der Apartheid Benachteiligten bislang beständig einer Politik der Freiwilligkeit $^{3}$ den Vorzug gegeben hatte: Farmland wurde bislang zu (auf Augenhöhe) ausgehandelten Preisen vom Staat aufgekauft, um anschließend subventioniert vom Staat neu übereignet $\mathrm{zu}$ werden. Dies geschah allerdings wegen eines geringen Haushalts und wegen nur mäßiger Bereitwilligkeit auf Verkäuferseite nicht in großem Stil. Der nunmehr anscheinend einsetzende Politikwechsel ${ }^{4}$ hin zur Enteignung ${ }^{5}$ als Mittel zur beschleunigten Implementierung der Landreform weckt Hoffnungen und Ängste.

$\mathrm{Zu}$ anderen Zwecken (etwa Verkehrswegeplanung) wurde das Institut der Enteignung schon vorher angewandt.

Dies ist prinzipiell in Art. 1, 2, 7 ff. der endgültigen Verfassung (Constitution of the Republic of South Africa 1996; im Folgenden VerfRSA) verankert und so entspricht es der Verfassungswirklichkeit. Der Verfassungstext ist abrufbar unter http://www.info.gov.za/documents/. Sog. ,policy of willing seller / willing buyer“, Prinzip von Angebot und Nachfrage. Vgl. auch Staatspräsident T. Mbeki, State of the Nation Address vom 3.2.2006, in der er mit knappen Worten eine Überprüfung der bisherigen Politik durch das Landministerium ankündigt.

5 Man versteht unter Enteignung im Allgemeinen den vollständigen oder teilweisen Entzug konkreter eigentumsfähiger Rechtspositionen gegen den Willen des Eigentümers final zur Erfüllung bestimmter Gemeinwohlaufgaben. Umfassend H.-J. Papier in: Maunz/Dürig, Grundgesetz, Kommentar, Rn. 523 ff. zu Art. 14. Vgl. auch BVerfGE 52, 1, 27. Zum südafrikanischen Recht Constitutional Court, CCT 9/97 - „Harksen v Lane NO and Others“, 16 ff. 
Zur Bewertung der Ereignisse müssen staatliche Landkäufe und -enteignungen zunächst vor dem Hintergrund des Projekts Landreform als Ganzes in seiner gesellschaftlichen Dimension, seiner Geschichte und den damit verbundenen Konflikten betrachtet werden.

Die Landreform lässt sich unterteilen in erstens das allgemeine Bestreben nach einer gerechten Umverteilung des Landes hinsichtlich des Eigentums (Redistribution), zweitens eine Absicherung von unsicheren Besitz- und Nutzungsrechten (Tenure Reform) und drittens die konkrete Wiedergutmachung von konkretem Apartheidsunrecht ${ }^{6}$ (Restitution). ${ }^{7}$ Die Landreform hat wegen ihrer besonderen Bedeutung für das südafrikanische Volk in diesen drei Facetten Verfassungsrang, minutiös festgeschrieben in Art. 25 (dazu unten). Allgemeiner gefasst untermauert dieses Anliegen zudem die Präambel der VerfRSA, die nicht nur eine Rückgängigmachung der eingetretenen gesellschaftlichen Spaltung avisiert, sondern sich auch zum Streben nach Verbesserung der Lebensqualität aller Staatsbürger Südafrikas und nach sozialer Gerechtigkeit bekennt. Denn zu all dem ist ausgewogen verteilter Landbesitz der Schlüssel.

Die weithin anerkannte Notwendigkeit der Landreform in Südafrika erklärt sich aus der Apartheidspolitik in der Geschichte. Insbesondere infolge der Regelungen im Natives' Land Act aus dem Jahr 1913 kurz nach der Gründung der Südafrikanischen Union, der Gebietszuweisungen für bestimmte ethnische Gruppen und Erwerbsverbote in anderen Gebieten vorsah, entstand eine sehr ungleiche Verteilung des Landes. Die weißen Nachfahren europäisch-stämmiger Siedler, die lediglich $10 \%$ der heutigen Bevölkerung stellen, waren am Ende der Ära der Apartheid Eigentümer von $87 \%$ der landwirtschaftlich nutzbaren Fläche; die übrigen $13 \%$ jener Fläche waren der überwältigenden, nicht-weißen Mehrheit zugewiesen und unterlagen der Verstaatlichung durch den Land Distribution Act 1936. Im Zuge der Rassentrennung wurden Schwarze in sog. Townships und Homelands vertrieben, sog. „black spots“ innerhalb des weißen Territoriums aufgelöst. ${ }^{8}$ Im Ergebnis stehen rund 60.000 weiße Großfarmen vielen Millionen von Nicht-Weißen gegenüber, die bei Überwindung des Apartheidsrégimes und dem Beginn des „,neuen Südafrikas“ 1994 landund mittellos waren.

Zu dessen völkerrechtlicher Ächtung siehe nur die "UN International Convention on the Suppression and Punishment of the Crime of Apartheid" von 1973, aber auch die Diskriminierungsverbote in Art. 2 der Allgemeinen Erklärung der Menschenrechte von 1948 und Art. 26 des Internationalen Paktes für bürgerliche und politische Rechte von 1966 sowie das Übereinkommen zur Beseitigung jeder Form von Rassendiskriminierung von 1966. M. Herdegen, Völkerrecht, 4. Aufl. 2005, § 47 Rn. 3, zählt Rassendiskriminierungsverbote auch zum Völkergewohnheitsrecht. C. G. van der Merwe/ J. E. du Plessis (Hrsg.), Introduction to the Law of South Africa, 2004, 240. im Einzelnen siehe S. Preißler, Die Rückgabe von Landrechten in Südafrika, 1998, 7 ff., 31 ff. 
Angesichts dieses Missverhältnisses empfindet die Bevölkerungsmehrheit die bisher praktizierte, auf Freiwilligkeit basierende Landreformpolitik als zu schleppend. ${ }^{9}$ Sie verlangt nach Beschleunigung und weitergehender Umverteilung; die anhaltende Diskrepanz zwischen Arm und Reich schlägt sich in den hohen Kriminalitätsraten nieder. Kritisiert wird zum Beispiel auch, dass die staatlichen Stellen zum Aufkauf von Agrarland im letzten Jahrzehnt wegen Unterbesetzung und nicht adäquat ausgebildetem Personal zu wenig effektiv gewesen seien. ${ }^{10}$ Insgesamt wird über die Art und Weise der Änderung des status quo diskutiert; ein breites Spektrum von Maßnahmen ist im Gespräch. ${ }^{11}$ Dass nicht so weiter gemacht werden kann wie bisher, wurde auf dem jüngsten Gipfeltreffen zur Landfrage unter Beteiligung sämtlicher Interessengruppen deutlich. ${ }^{12}$ Die Politik tendiert insbesondere vor Wahlen auf lokaler Ebene - in stärker werdendem Ausmaß in dieselbe Richtung, zumal in einem Wiederaufbau- und Entwicklungsprogramm ${ }^{13}$ des seit 1994 ununterbrochen mit einer knappen Zweidrittelmehrheit in der Nationalversammlung regierenden African National Congress (ANC) das noch immer als unumstößlich ${ }^{14}$ angesehene Ziel formuliert wurde, bis zum Jahr $201430 \%$ der Umverteilung der genannten $87 \%$ Farmland zu erreichen, von denen bisher - der jetzt in Frage gestellten Politik der Freiwilligkeit geschuldet -nach über der Hälfte der Zeit nur bloße $3 \%$ erreicht sind. ${ }^{15}$ Ein gesetzgeberischer Beschleunigungsversuch wurde mit einer Verschärfung des Enteignungsrechts im Jahre 2004 (im Einzelnen unten) unternommen, jedoch ist die Verwaltung in der Ausnutzung zu zurückhaltend gewesen.

Das schnelle Streben nach Veränderung begründet einen Interessenkonflikt, dessen Lösung Aufgabe von Politik und Recht ist, denn auch die Interessen der Großfarmer und andere

N. Göler von Ravensburg, Südafrikanisches Bodenrecht zwischen Verteilungsgerechtigkeit und Wirtschaftlichkeit, Recht in Afrika 2001, 17 (36).

10

D. Robertson, http://www.voanews.com vom 23.9.2005.

Zu möglichen Lösungswegen unten. Vgl. hier auf der einen Seite nur die Ansicht der (allerdings wenig bedeutenden) kommunistischen Partei, Veröffentlichung vom 22.9.2005, http://www.sacp. org.za: Die Entschädigungen sollen a priori vom Staat festgesetzt und es solle nunmehr flächendeckend enteignet werden. Die andere Extremposition (Landreform nur über den Markt) vertreten manche Großfarmer, vgl. das Position Paper der Transvaal Agriculture Union bei http://land.pwv. gov.za/Land_Summit/.

Information zu den Beteiligten und Zusammenfassung der Ergebnisse im Einzelnen vgl. G. Pabst, http://www.capetown-online.com vom 8.10.2005. Vgl. auch Dept. of Land Affairs, Land and Agrarian Reform in S.A.: An Overview in preparation for the Land Summit, $27^{\text {th }}-31^{\text {st }}$ July 2005.

13

„Reconstruction and Development Programme“ von 1994 (http://www.polity.org.za/html/ govdocs/rdp/rdp.html). Zusammenfassend K. Koppe, Wiedergutmachung für die Opfer von Menschenrechtsverletzungen in Südafrika, 2005, 193. Siehe auch Department of Land Affairs, Strategic Plan 2005-2010, März 2005, 8.

Landministerin Didiza wird zitiert mit: „Policy, and not the deadline, will have to change.“ ( $B$. 
öffentliche Interessen liegen in der Waagschale. Zu nennen sind primär der (Eigentums)Bestandsschutz, der Grundsatz des Vertrauensschutzes, den auch das südafrikanische Verfassungsrecht kennt, ${ }^{16}$ und damit die Tatsache, dass die Farmerfamilien die Liegenschaften z. T. auch schon seit mehreren Generationen bewirtschaften und ihrerseits, mitunter durch erhebliche Investitionen, eine enge Verbindung zu ihrem Lebenswerk aufgebaut haben. Die viel zitierten beiden ökonomischen Argumente im Allgemeininteresse Südafrikas dürfen bei der Konfliktlösung ebenfalls nicht außer Acht gelassen werden: ${ }^{17}$ Zum einen verfügen die bestehenden Farmbetriebe über agrartechnisches Know-how und adäquate Sachmittel, und sie sind auch wegen ihres Zuschnitts in der Lage, den Grund und Boden effektiv, im Rahmen der Globalisierung wettbewerbsfähig und zur Gewährleistung größtmöglicher Nahrungsmittelsicherheit zu nutzen - dies alles wird als zweifelhaft angesehen, wenn Land in viele kleine Abschnitte zersplittert und von vielen zunächst branchenfernen Neueigentümern bewirtschaftet werden würde. Zum anderen kann das Investitionsklima im Land durch die entstehende Rechtsunsicherheit nach Enteignungen, insbesondere wenn der Eindruck von Willkür entsteht, in größerem Stil leiden. Der Konflikt ist also auch einer im Spannungsverhältnis zwischen Armutsbekämpfung und Funktionieren der Wirtschaft.

Wegen der nicht unwesentlichen auf dem Spiel stehenden Werte, der existenziellen Bedeutung von Land, wegen der noch tiefen Gräben zwischen den unterschiedlichen Bevölkerungsteilen Südafrikas und der einschneidenden Ungerechtigkeiten in der Vergangenheit wird die Debatte bisweilen emotional geführt. ${ }^{18}$ Im Allgemeinen herrscht in Südafrika jedoch der Wille vor, auch auf dem Gebiet der Landreform die weise und moderate Politik der Wahrheit und Versöhnung fortzusetzen, die einem zu radikalen Vorgehen, das wiederum neue Narben hinterlassen kann, entgegensteht.

Eine weitere Schwierigkeit bereitet in vielen Fällen die historische Rekonstruktion der Frage, unter welchen Umständen genau welche Abschnitte Land einst von weißen Siedlern erworben worden waren. ${ }^{19}$ Diese Umstände sind relevant für die rechtliche Entscheidung,

I. Currie/ J. de Waal (Hrsg.), The New Constitutional and Administrative Law, Vol. I: Constitutional Law, 2001, 77, 81: die Inhalte des deutschen Rechtstaatsprinzips liegen der südafrikanischen rule of law zugrunde; auch I. M. Rautenbach/ E. F. J. Malherbe, Constitutional Law, 2. Aufl. 1996, 280 f. erwähnen ,legal certainty“ als Bestandteil der VerfRSA.

Vgl. nur J.-P. Kapp in: Neue Zürcher Zeitung vom 21.10.2005, http://www.nzz.ch.

18

Etwa wenn auf der einen Seite erklärt wird, es gehe den Anspruchstellern nur um persönliche Bereicherung, und auf der anderen Seite, die Farmer wünschen sich die Zeiten der Apartheid zurück. Manche Farmer sprechen gar von Landdiebstahl, Plünderung, Belästigung und drohen notfalls mit bewaffnetem Kampf, vgl. M. le Roux in: The Star vom 8.9.2005, http://www.thestar. co.za.

19

Es gab es keine einheitliche Politik der Kolonialisten hinsichtlich der angetroffenen Stämme. Ungewissheit besteht zudem wegen manchen Wanderungsbewegungen von Bevölkerungsteilen 
welcher Aspekt der Landreform verfolgt wird - Restitution an vormalige, rassistisch diskriminierte Eigentümer oder generelle Umverteilung von Landbesitz. Geschah z. B. keine Aneignung aufgrund rassendiskriminierender Gesetze (und war sie möglicherweise nach heutiger Rechtslage in jeder Hinsicht verfassungsgemäß), sind andere, naturgemäß höherschwellige Rechtsgrundlagen für eine Enteignung einschlägig. ${ }^{20}$

Die Betrachtung der südafrikanischen Enteignungsverfahren ist schließlich auch und gerade vor dem Hintergrund der jüngeren Landpolitik der Nachbarländer interessant, in denen ein ähnliches, (kolonial-)historisch bedingtes Wohlstandsgefälle zwischen den Nachfahren europäisch-stämmiger Siedler und der schwarzen Bevölkerungsmehrheit besteht:

Abschreckendes Beispiel ist insoweit das von Präsident Mugabe autokratisch geführte Simbabwe $^{21}$, wo es seit $2001^{22}$ auf der Grundlage eines Land Acquisition Acts und unter den Schlagworten „fast track resettlement"/,land grab“ zu zahlreichen Enteignungen weißer Farmen gekommen ist, die entschädigungslos ${ }^{23}$ und faktisch ohne die Möglichkeit zu wirksamen Rechtsbehelfen erfolgten und begleitet waren von Besetzungen, Räumungen, Gewalt und Tod, und bei denen Mugabe insbesondere seine politischen Weggefährten begünstigte. ${ }^{24}$ Die ehemals knapp 4.500 weißen Farmen sind nunmehr auf wenige hundert reduziert. Anerkanntermaßen war die willkürliche Zerschlagung funktionierender wirtschaftlicher Einheiten neben der Dürre Grund für die derzeitige ökonomische Krise und den faktischen Bankrott des Staates. Als Mugabe zuletzt dem südafrikanischen Politikwandel applaudierte, hat Präsident Mbeki (nach einer zunächst missverständlichen Äußerung der Vizepräsidentin Mlambo-Ngcuka) ${ }^{25}$ entschieden zurückgewiesen, dass nunmehr dem Beispiel Simbabwes gefolgt werde. ${ }^{26}$ Es soll hier herausgestellt werden, dass die Situation

(schwarz und weiß), mangelhafter Landvermessung und Dokumentation von Erwerbsvorgängen. Auch die Umstände der Enteignung/Vertreibung von Schwarzen zwischen 1913 und 1948 variierten stark.

Constitutional Court, CCT 19/03 - "Alexkor Ltd. v The Richtersveld Community and Others" mit dem Streitpunkt, ob eine historische Enteignung wegen Diamantenfunden auf einem Grundstück rassistisch diskriminierend i.S.d. Restitution of Land Rights Act war.

Das demokratische und rechtsstaatliche Defizit von dessen Verfassung im Einzelnen darlegend $J$. Makumbe in: Sichone (Hrsg.), The State and Constitutionalism in Southern Africa, 1998, $65 \mathrm{ff}$.

J. Makumbe, a.a.O., 69: Bereits durch Act 11, 1990, wurde Land für das „Resettlement“ ausgewiesen und Rechtsstreitigkeiten über die Höhe einer Entschädigung wurden ausgeschlossen.

Entschädigung gab es allenfalls für Investitionen auf dem Grund und Boden, nicht für diesen selbst, und z. T. auch nur in Regierungsschuldverschreibungen.

Mit einem tabellarischen Abriss der simbabwischen Landreformgeschichte http://www.auswaertiges-amt.de/www/de/laenderinfos. Siehe auch T. A. S. Bowyer-Bower/ C. Stoneman (Hrsg.), Land Reform in Zimbabwe: Constraints and Prospects, 2002.

26

G. Pabst, a.a.O.

SAPA, Mail \& Guardian Online vom 27.10.2005, http://www.mg.co.za. 
in Südafrika keineswegs mit der in Simbabwe verglichen werden kann, wie man bei flüchtiger Lektüre der Schlagzeilen befürchten könnte.

In der demokratischen Republik Namibia stehen zum Jahreswechsel wegen wachsenden Unmuts im Volk ebenfalls Enteignungen mancher Großfarmen auch deutschstämmiger Bürger an, ${ }^{27}$ was dort ebenfalls Unsicherheit auf Seiten der Farmer, Erwartungen auf Seiten der landlosen Bevölkerungsmehrheit auslöst. ${ }^{28}$ Die Entschädigungshöhe richtet sich nach dem Marktwert. Die viel gelobte Verfassung von Namibia ${ }^{29}$ lässt die Landfrage allerdings im Gegensatz zur VerfRSA bei ihrer Formulierung des Eigentums- und Enteignungsrechts in Art. 16 gänzlich außen vor und erwähnt sie auch sonst mit keinem Wort ausdrücklich, ${ }^{30}$ wenngleich diesbezüglich in diesem Staat seit der Unabhängigkeit 1990 ein ähnlicher Problemdruck wie in Südafrika herrscht. ${ }^{31}$ Von offizieller Seite wird versichert, dass sämtliche Maßnahmen zur Landreform auf dem Boden von Recht und Gesetz (d. h. auch unter Beachtung der allgemeinen verfassungs- und grundrechtlichen Anforderungen an eine Enteignung: „öffentliches Interesse“ und „gerechte Entschädigung“, Art. 16 Abs. 2 VerfNam) durchgeführt werden, ${ }^{32}$ woran bis jetzt in der Realität (trotz manch nebulöser und manch radikaler Rhetorik von Politikern) ${ }^{33}$ keine Zweifel bestehen. Die weitere Entwicklung in Namibia bleibt spannend, und es ist abzuwarten, wie Exekutive und Judikative die bestehenden Spielräume der Normen in Zukunft nutzen.

Zurückkehrend zur Situation in Südafrika sollen im Folgenden zunächst der den laufenden Enteignungsverfahren zugrunde liegende und zu diesem Aufsatz Anlass gebende Sachverhalt kurz exemplarisch geschildert und die rechtlichen Grundlagen zum südafrikanischen

Ein Sachverhalt ist nachzulesen auf http://news.bbc.co.uk/2/hi/africa/4207748.stm und Mail \& Guardian Online vom 17.11.2000, (Fn. 27).

Eingehend zu den namibischen Besonderheiten H. Sippel, Landfrage und Bodenreform in Namibia, VRÜ 2001, 292 ff., der auch die dortige Bodenrechtsgeschichte aufarbeitet.

Constitution of the Republic of Namibia 1990 (VerfNam). Zu Entstehungsgeschichte und Inhalt W. Müller, Verfassung von Namibia: Ethnische Vielfalt und Elemente eines Minderheitenschutzes, 1992; kritisch H. Melber, in: Sichone (Fn. 21), 15 ff.

Auch nicht im ausgedehnten Katalog der Staatsziele des Art. 95 VerfNam (,Promotion of the Welfare of the People"). Eine Besonderheit im namibischen Recht ist jedoch, dass der namibische Gesetzgeber ausdrücklich ermächtigt wird, Ausländer im Erwerb von Eigentum zu beschränken (womit die Möglichkeiten von Einheimischen zum sukzessiven Landerwerb zu geringeren Preisen gesteigert werden). Begleitend zu einer nur generellen Möglichkeit der Enteignung ebnet ferner Art. 23 Abs. 2 VerfNam verfassungsrechtlich lediglich den Weg für eine Gesetzgebung zu einer unspezifischen Affirmative Action der Diskriminierungsopfer der Apartheidsvergangenheit in allen Lebensbereichen.

80 Prozent des kommerziellen Farmlands befinden sich in der Hand von Weißen. Guardian Online vom 28. und 29.11.2005, (Fn. 26).

Vgl. H. Sippel, (Fn. 28), 293. 
Recht der Enteignung und der Landrestitution aus der Perspektive des deutschen Rechts dargestellt werden. Anschließend geht es um die Subsumtion des Sachverhalts unter das Recht und eine Bewertung der Landreform.

\section{B. $\quad$ Ein Sachverhalt ${ }^{34}$}

Objekt des ersten Enteignungsverfahrens ist die etwa 500 ha große Schaf- und Viehzuchtfarm des Viehwirtschaftlers Hannes Visser in der an Namibia grenzenden Provinz Nordwest $200 \mathrm{~km}$ westlich von Johannesburg. Nach Medienangaben sind über zwei Jahre Verkaufsverhandlungen mit dem Staat geführt worden, die jedoch ohne Erfolg geblieben waren, weil Visser wegen der vom ihm getätigten Investitionen in den angeschlossenen Fleischverarbeitungsbetrieb einen Kaufpreis von 3 Mio. Rand (ca. $380.000 €$ ) verlangte, der Staat aber unter Berufung auf ein unabhängiges Wertgutachten nur 1,75 Mio. Rand (ca. $230.000 €$ ) bezahlen wollte, so dass das Verfahren stockte. Antragstellerin ist die Erbengemeinschaft nach Molamu, der bis 1942 Eigentümer war. Sie macht einen Anspruch auf Restitution geltend, weil Molamu durch einen erzwungenen Verkauf im Zuge der Durchsetzung von Rassentrennungsgesetzen aus dem Eigentum gesetzt worden sei. Visser hingegen trägt neben seiner abweichenden Ansicht zum Grundstückswert vor, Molamu habe das Land 1942 zum Marktpreis rechtsgültig veräußert und sein Vater habe es seinerseits 1968 erworben - es handle sich also nicht um einen Restitutionsfall. Nach der Ankündigung der Enteignungsabsicht durch die Land Claims Commission, die dem Landministerium (Landministerin Didiza) unterstellt ist, hat Visser Gelegenheit zur Stellungnahme gehabt. Ein Ergebnis wird in den nächsten Wochen erwartet. Visser hat bereits angekündigt, den erwarteten Beschluss zur Enteignung gegen ihn gerichtlich überprüfen lassen zu wollen.

Neben der Visser-Farm hat die Land Claims Commission bereits fünf weiteren Farmen in Nordwest mit jeweils ähnlichen Tatsachenhintergründen ihre Absicht mitgeteilt, dass sie Enteignungsverfahren einleiten werde. ${ }^{35}$

\section{Das südafrikanische Recht der Enteignung aus deutscher Perspektive}

Welche Rolle spielt das Recht in der Landreform und den konkreten Enteignungsvorhaben und wie ordnet es die damit zusammenhängenden Fragen?

Nachzulesen bei F. Peete in: The Mercury vom 23.9.2005, http://www.themercury.co.za; $B$. Boyle, (Fn. 14); aus der internationalen Presse: D. Robertson, (Fn. 10); M. Wines in: The New 35 York Times vom 27.9.2005, http://www.nytimes.com; J.-P. Kapp, (Fn. 17).

M. le Roux in: Cape Times vom 28.9.2005, http://www.capetimes.com. 


\section{Verfassungsrecht}

Wegen des Vorrangs ${ }^{36}$ der Verfassung ist im Folgenden zunächst der verfassungsrechtliche Rahmen abzustecken, in dem sich das Eigentums- und das Enteignungsrecht in Südafrika bewegen. ${ }^{37}$ Der Rahmen steht fest, denn die Hürden für eine Verfassungsänderung sind hoch und nur in einem breiten Mehrparteienkonsens erreichbar. ${ }^{38}$ Die neun Absätze umfassende Regelung des Art. 25 VerfRSA („Property“) als Teil des Zweiten Kapitels („Bill of Rights“) soll kurz dargestellt werden. Dessen Regelungsgehalt ist dem deutschen Grundgesetzartikel 14 ähnlich, ${ }^{39}$ weist aber auch entscheidende, durch die Vergangenheit Südafrikas bedingte Unterschiede auf; insbesondere ist die VerfRSA insoweit wegen der Problematik der Landreform ausführlicher. ${ }^{40}$

Während in der südafrikanischen Interimsverfassung ${ }^{41}$ von 1994 Eigentumsgrundrecht (Art. 28) und die Bestimmungen zur Landfrage (Art. $121 \mathrm{ff}$.) noch systematisch getrennt waren, führt die endgültige Verfassung von 1996 diese Elemente, weil sachlich eng miteinander verwoben, mit abgewandeltem Inhalt zusammen. Art. 25 VerfRSA wird als die wohl kontroverseste Bestimmung der Verfassung angesehen. ${ }^{42}$ Sie markiert Kompromiss und Grundkonsens der zwei an den Verfassungsverhandlungen teilnehmenden Hauptgruppierungen: Die damalige National Party (NP) setzte sich für einen größtmöglichen Schutz der Besitzstände ihrer weißen Klientel ein, der ANC für eine friedliche Rückgängigmachung der Apartheidsfolgen in jeder Hinsicht. ${ }^{43}$

36

37

38

39

40

43

Art. $1 \mathrm{c}$ ) und 2 VerfRSA.

Die Strukturen der VerfRSA als Ganzes fasst kurz zusammen U. Häußler, JA 1997, 995.

Dies trotz der überwältigen, absoluten Mehrheit des ANC; vgl. die nach Änderungsgegenstand abgestuften Quoren in beiden Kammern und das Verfahren gemäß Art. 74 Abs. 1-9 VerfRSA.

Und sogar von ihm während der Verfassungsgebung beeinflusst, vgl. J. Fedtke, Die Rezeption von Verfassungsrecht, 2000, 314 ff. m.w.N.

Auch (der praktisch unbedeutende) Art. 15 GG befasst sich in Deutschland mit Eigentum und dessen Entzug, allerdings geht es dort um Sozialisierung, d. h. die Überführung in Gemeineigentum, also gerade nicht um die hier interessierende Umverteilung unter Privaten, vgl. B.-O. Bryde in: vMünch/Kunig, Grundgesetzkommentar Bd. 1, 5. Aufl. 2000, Rn. 1, 7 f. zu Art. 15.

Den südafrikanischen Verfassungsgebungsprozess von 1990 über CODESA, Multi-Party Negotiating Process, das Government of National Unity, die 34 unverletzlichen Verfassungsprinzipien bis zum Certification Judgement des Constitutional Court zusammenfassend statt vieler J. Fedtke, (Fn. 39), 58 ff., und T. M. Grupp, Südafrikas neue Verfassung, 1999, $17 \mathrm{ff}$.

G. E. Devenish, A Commentary on the South African Constitution, 1998, 71 m.w.N.; J. Zimmerman, Property on the Line: Is an Expropriation-Centred Land Reform Constitutionally Permissible?, SALJ 2005, 378 (383 ff.): Manche Gruppierung lehnten aus Angst vor einer Zementierung der Besitzstände eine Eigentumsgarantie sogar gänzlich ab.

Eingehend auf die Debatten bei Schaffung der Vorschrift A. M. Gross, The Dilemma of Property Rights in Ethnic Land Regimes: Israel and South Africa Compared, SALJ 2004, 448 (449 f.). 
Wie in Deutschland fällt auch in Südafrika nicht nur das bürgerlich-rechtliche bewegliche und unbewegliche Sacheigentum in den Schutzbereich der Eigentumsgarantie, sondern jede wohlerworbene Rechtsposition des einfachen Rechts. ${ }^{44}$

Hier wie dort wird zunächst qualitativ zwischen zwei Arten von Eigentumseingriffen mit verschiedenen Rechtfertigungsanforderungen unterschieden: den Inhalts- und Schrankenbestimmungen einerseits und Enteignungen andererseits (,deprivation“ und ,expropriation“). ${ }^{45}$ Art. 25 Abs. 1 VerfRSA bezieht sich auf die „,deprivation“, welche nur durch ein allgemeines Gesetz, das keinen Raum für Willkür lässt, ${ }^{46}$ zulässig ist. ${ }^{47}$

Die Abs. 2-4 befassen sich mit der hier im Mittelpunkt stehenden „expropriation“. ${ }^{48}$ Abs. 2 stellt die Zulässigkeitsvoraussetzungen für eine Enteignung auf: Enteignet werden darf nur aufgrund eines allgemeinen Gesetzes zu einem öffentlichen Zweck bzw. in öffentlichem Interesse und zum Schutz des zu enteignenden Adressaten nur gegen eine Entschädigung, die entweder mit diesem vereinbart wurde oder von einem Gericht beschlossen oder gebilligt wurde. 49

Abs. 3 gestaltet das Entschädigungsrecht detailliert aus: Entschädigungen müssen danach gerecht und billig sein sowie unter Abwägung aller relevanten Umstände einen billigen Ausgleich zwischen dem öffentlichen Interesse und dem Interesse des Betroffenen gewährleisten. Den Begriff der hier abzuwägenden „Umstände“ soll der beispielhafte Katalog im Anschluss erhellen; zu beachten sind die gegenwärtige Nutzungsart des Eigentums, die Historie von Erwerb und Nutzung, der Marktwert, das Ausmaß bisheriger staatli-

Art. 25 Abs. 4 b) VerfRSA stellt klar, dass jedenfalls nicht nur Eigentum an Grund und Boden erfasst sein soll, obwohl der Artikel ansonsten stark auf die Landfrage ausgerichtet ist. Zum südafrikanischen Eigentumsbegriff G. E. Devenish, (Fn. 42), 71. Vgl. zum deutschen nur BVerfGE $89,1,7$.

Zum Verhältnis der beiden Eingriffe in der Rechtsprechung des Verfassungsgerichts A. J. van der Walt, Striving for a Better Interpretation - A Critical Reflection on the Constitutional Court's Harksen and FNB Decisions on the Property Clause, SALJ 2004, 854 ff.: Während bei „Harksen“ die Eingriffe noch trennscharf als alia behandelt werden, wird in ,First National Bank“ (CCT 19/01) die Enteignung als Sonderfall der Inhalts- und Schrankenbestimmung angesehen, für die auch sämtliche Anforderung an diese (insbesondere keine ,arbitrariness“) zu gelten haben.

Das Willkürverbot ist hier deklaratorisch, da es bereits vom Rechtsstaatsprinzip umfasst ist, I. Currie/ J. de Waal (Hrsg.), (Fn. 16), 79 f. Zu den Prinzipien ,rule of law“ und „,constitutional state“ siehe I. M. Rautenbach/ E. F. J. Malherbe, Constitutional Law, 2. Aufl. 1996, 279-282.

Näher I. M. Rautenbach/E. F. J. Malherbe, (Fn. 46), 329: Eine (verfassungsrechtlich zu rechtfertigende) „Deprivation“ sei schon jede Einschränkung der freien Verfügbarkeit über das Eigentum.

In Deutschland ist für Enteignungen Art. 14 Abs. 3 GG Regelungsort, in dem es heißt, eine Enteignung ist nur zum Wohle der Allgemeinheit und nur durch oder aufgrund eines Gesetzes mit dem Junktim einer gesetzlichen Entschädigungsklausel zulässig. 
cher Förderung von Erwerb und Investitionen in der Vergangenheit sowie schließlich der Zweck der Enteignung. Weist also beispielsweise der einstige Erwerb von Land durch einen Siedler den Makel auf, dass er damit entweder selbst jemandem Unrecht getan hat oder er nur aufgrund eines diskriminierenden Gesetzes oder einer diskriminierenden Verwaltungspraxis beim Landerwerb zum Zuge kam, so kann die Entschädigung auch für den Rechtsnachfolger den Marktwert, der bewusst nur als einer unter mehreren Faktoren genannt ist, weitaus unterschreiten. 50

In den ersten drei Absätzen des Art. 25 wird das Recht der Enteignung aber noch losgelöst von der Frage der Landreform behandelt; die Enteignungen können jeden Gegenstand betreffen. Die Systematik geht vom Allgemeinen zum Speziellen. Der bereits angeklungene Verfassungsrang der Landreform in Südafrika manifestiert sich namentlich nun zum ersten Mal in der Auslegungsregel des Art. 25 Abs. 4 a) VerfRSA zum Begriff des öffentlichen Interesses. Die Auslegungsregel gibt zwingend vor, dass das öffentliche Interesse als Enteignungsvoraussetzung (Abs. 2) den Willen der Nation zu einer Landreform enthalte und den Willen zu allen Reformen, die einen gleichen Zugang zu den „Naturressourcen“ Südafrikas für alle Südafrikaner eröffnen sollen. Damit macht der Verfassungsgeber deutlich, dass die (erste Facette der) Landreform im Sinne einer grundsätzlichen Umverteilung Staatsziel ist. ${ }^{51}$

Bei der Frage, ob ein „öffentliches Interesse“ an der Landreform vorliegt, und bei den „Umständen“ zur Entschädigungsbemessung sind im übrigen auch weitere Verfassungswerte heranzuziehen, insbesondere die sozio-ökonomischen Grundrechte in Art. 24 ff. i. V. m. Art. 7 Abs. 2 VerfRSA, denen zu Wirksamkeit auch über das Instrument der Enteignung verholfen werden kann. ${ }^{52}$ Über die Festlegung des Abs. 4 a) hinausgehend (und abstrahiert vom Recht der Enteignung zu sehen) stellt Art. 25 Abs. 5 VerfRSA dann sogar eine allgemeine Handlungspflicht für staatliche Organe - insbesondere den Gesetzgeber ${ }^{53}$ - auf, im Rahmen ihrer Möglichkeiten proaktiv vernünftige Bedingungen dafür zu schaffen, dass Bürger in billigem Umfang Zugang zu Land erhalten. Diese Aussage bekräftigt das staatli-

Nach J. Zimmerman, (Fn. 42), 405 ff., seien wegen historischer, norm- und verfassungssystematischer Argumente die Entschädigungen im Rahmen der Landreform generell geringer als zu jedem anderem Zweck.

51 J. Zimmerman, (Fn. 42), 383: ,special constitutional priority“ von Landreform-Enteignungen. Zur Bedeutung von Staatszielbestimmungen und insbesondere jenen im GG C. Degenhart, Staatsrecht I: Staatsorganisationsrecht, 21. Aufl. 2005, § 6; T. Würtenberger/ R. Zippelius, Deutsches Staatsrecht, 31. Aufl. 2005, § 13.

J. Zimmerman, (Fn. 42), 395. Nach Art. 39 VerfRSA sind im Übrigen auch internationale Rechtsstandards zu berücksichtigen.

National Assembly (Art. 46 ff. VerfRSA) und National Council of Provinces (Art. 60 ff.) bilden die Legislative auf nationaler Ebene. Zum Gesetzgebungsverfahren vgl. Art. 73 ff.; zu den Kompentenzen die Anhänge 4, 5. 
che Interesse an Umverteilung noch und kann für die Verhältnismäßigkeit von in das Eigentum eingreifenden Gesetzen von Bedeutung sein. Es handelt sich dabei allerdings um objektives, d. h. nicht individuell einklagbares Recht. ${ }^{54}$

Der folgende Abs. 6 beschäftigt sich mit der nächsten Facette der Landreform, die den Landbesitz, also die Formen der tatsächlichen Sachherrschaft, betrifft (Tenure Reform). Der Absatz verbürgt zusätzlich zum obigen Eigentumsrecht ein Grundrecht auf Rechtssicherheit für natürliche Personen und für Stammesgemeinschaften („Communities“) ${ }^{55}$, deren Landbesitzrechte in der Vergangenheit aufgrund von (heute unwirksamen) rassendiskriminierenden Gesetzen oder Praktiken begründet worden waren. Wegen der rigiden, auf Trennung ausgerichteten Rechtslage sind während der

Apartheid viele illegale Besitz- und Nutzungsverhältnisse mit der armen, schwarzen Landbevölkerung entstanden, ${ }^{56}$ die nunmehr nicht als rechtsgrundlose Besitzer der Vindikation, also der Vertreibung, durch die Eigentümer ausgesetzt sein sollen. Die Adressaten des Grundrechts haben deshalb von Verfassungs wegen Anspruch entweder auf die rechtssichere Neubegründung ihres Landnutzungsrechts nach geltendem Recht oder auf die Zuerkennung einer vergleichbaren Ersatzleistung. Zur Regelung im einzelnen ist gemäß Abs. 9 der nationale Gesetzgeber berufen, der hierzu den Land Reform (Labour Tenants) Act $1996^{57}$ und den Extension of Security of Tenure Act $1997^{58}$ beschlossen hat. ${ }^{59}$

Nachdem die Landumverteilung in puncto Enteignung und Ermöglichung von Zugang zum Landeigentum und die Reform der Besitzrechte in den Absätzen 4, 5 und 6 thematisiert worden sind, greift die VerfRSA im Anschluss daran mit Art. 25 Abs. 7 auch die letzte Facette der Landreform - Restitution - direkt auf: Natürliche Personen und Stammesge-

54

55

56

57

58

59

Zu diesem Komplex zählen ferner der Upgrading of Land Tenure Rights Act 1996, der Interim Protection of Informal Rights Act und der Prevention of Illegal Eviction From and Unlawful Occupation of Land Act 1998. Zu weiteren Elementen der „Tenure Reform“ vgl. auch Department of Land Affairs, Strategic Plan 2005-2010, März 2005, 6. Auch in Deutschland bestand im Übrigen nach dem Beitritt der neuen Länder ein Bedarf an zivilrechtlichen Übergangsregelungen für Nutzungsverhältnisse (vgl. die Gesetze zur Schuldrechtsanpassung und Sachenrechtsbereinigung). 
meinschaften, die nach dem 19. Juni 1913 (Inkrafttreten des erwähnten Natives’ Land Act) durch rassendiskriminierende Gesetze oder Praktiken aus dem Eigentum gesetzt worden sind, haben Anspruch auf Restitution oder einen der Billigkeit entsprechenden Ersatz. Die Verankerung der Restitution im neuen System soll einerseits der Wiedergutmachung dienen, aber auch im noch jungen Rechtsstaat für Vertrauen in die Eigentums- und Rechtsordnung und für Rechtsfrieden sorgen. Strukturpolitisch soll zudem wieder breiteren Schichten zu Arbeit und einer Existenzgrundlage verholfen werden. ${ }^{60}$ Das schon in der Vorgängervorschrift des Art. 121 Interimsverfassung dazu ermächtigte Parlament hat in Ausgestaltung dieses Regelungskomplexes den Restitution of Land Rights Act 1994 (dazu unten) erlassen. Abs. 8 schließlich stellt mit seiner Formulierung sicher, dass die Eigentumsgarantie zwar keinesfalls Reformen im Wege stehen darf, die darauf abzielen, die Ungerechtigkeiten der Apartheidszeit zu beseitigen. Er stellt aber auch sicher, dass dennoch jedwede Beschränkung des Eigentums der allgemeingültigen Schranken-Schranke ${ }^{61}$ des Art. 36 Abs. 1 VerfRSA („Limitation of Rights“) standhalten muss. Es handelt sich daher bei Abs. 8 zum einen um eine erneute (deklaratorische) Bekräftigung des Staatsziels Landreform, zum anderen um eine Kollisionsnorm hinsichtlich der Beschränkbarkeit in dem Sinne, dass mit Art. 36 eine weitere Schranken-Schranke kumulativ gilt, die nicht durch Art. 25 als lex specialis verdrängt wird. ${ }^{62}$ In Art. 36 finden sich z. B. das Verbot von Einzelfallgesetzen und genaue Richtlinien für eine Verhältnismäßigkeitsprüfung. ${ }^{63}$

\section{Einfaches Gesetzesrecht}

\section{Rechtslage in Südafrika}

Der Restitution of Land Rights Act 1994 (RLRA) ${ }^{64}$ formt den Gesetzgebungsauftrag aus Interimsverfassung und Art. 25 Abs. 7 VerfRSA verfassungsgemäß aus. Wenngleich der Anwendungsbereich weit gesteckt ist, soll das Gesetz auch bewirken, dass es nicht zu einer Totalrevision aller Maßnahmen des alten Systems kommt. ${ }^{65}$ Der RLRA kann Ansprüche

60

61

62

63

64

65

Zu den Funktionen der Restitution J. von Bismarck, (Fn. 55), 226.

Anforderungen, die ihrerseits an grundrechtsbeschränkende Gesetze gestellt werden. In Deutschland z. T. kodifiziert in Art. 19 Abs. 1, 2 GG; vgl. auch Art. 22 VerfNam und B. Pieroth/ B. Schlink, Grundrechte: Staatsrecht II, 21. Aufl. 2005, Rn. 274 ff.

Fraglich bleibt dennoch, was von der Verhältnismäßigkeitsprüfung im Rahmen des Art. 36 übrig bleibt, wenn die „Arbitrariness“-Prüfung schon so umfangreich angelegt ist wie beim „First National Bank"-Urteil, vgl. die berechtigte Kritik von A. J. van der Walt, (Fn. 45), 873.

Im Einzelnen I. Currie/ J. de Waal (Hrsg.), (Fn. 16), 338 ff.

Vgl. zu Entstehungsgeschichte und Anspruchsvoraussetzungen im Stand 1999 J. von Bismarck, (Fn. 55), 10 ff., $60 \mathrm{ff}$.

Vgl. J. von Bismarck, (Fn. 55), 234 ff.: „,offener Anwendungsbereich“. 
gegen den Staat Südafrika, nicht aber gegen Privatpersonen begründen. Der Staat muss tätig werden, um die Ansprüche auf Restitution zu erfüllen und ggf. seinerseits den Anspruchsinhalt, etwa ein zu übereignendes Grundstück, beschaffen.

Voraussetzung der formgerecht bis zum 30.4.1998 fristwahrenden Anträge ist, ${ }^{66}$ wie im Wesentlichen schon durch die VerfRSA vorgegeben, der Entzug eines Immobiliarsachenrechts nach dem 19.6.1913 aufgrund von rassendiskriminierenden Gesetzen oder Praktiken, ${ }^{67}$ für den keine ausreichende Entschädigung gewährt worden war, vgl. Art. 2 RLRA. Selbst ein redlicher Zwischenerwerb stellt in Südafrika keinen Ausschlussgrund ${ }^{68}$ dar, so dass dort grundsätzlich auch der Gutgläubige Adressat einer Enteignung werden kann. Die Rechtsfolge ist ggf. ein sehr weites Ermessen, wie die Abhilfe erfolgen soll. ${ }^{69}$

Die Prüfung, ob die Voraussetzungen für den Antrag gegeben sind, erfolgt anhand der einzureichenden Dokumente des Antragstellers ex officio durch die zuständige Land Claims Commission ${ }^{70}$ auf Provinzialebene ${ }^{71}$; die formelle Beweislast obliegt dem Antragsteller. Der Antrag ist grundsätzlich zur (weiteren) Ermittlung bei der Kommission zu stellen, die nach Mediation und Beilegungsversuchen, Information der Öffentlichkeit sowie der Prüfung von Einwendungen der Gegner und der Äußerungen der Exekutive der verschiedenen Ebenen letztlich (nötigenfalls) eine Entscheidung des eigens errichteten, regierungsunabhängigen Land Claims Courts ${ }^{72}$ einholen muss. Dem Antragsteller werden Auskunftsrechte gewährt und ihm werden Beratung und Unterstützung zuteil. Der Court ist dann in seiner Entscheidungsbandbreite nach Art. 35 RLRA von Gesetzes wegen sehr frei umfasst sind die Rückübertragung des streitgegenständlichen oder eines Ersatzgrundstücks, Entschädigungszahlung, Abhilfe auf andere Art und Weise (etwa durch bevorzugte Zuteilung staatlicher Hilfen ${ }^{73}$ und die Bestimmung aller Einzelheiten der Vollstreckung. ${ }^{74}$ Nach

Es sind derer rund 79.000 eingelegt worden, die bislang erst zu etwa $3 / 4$ erledigt sind.

Dieses Tatbestandsmerkmal wird teleologisch weit ausgelegt.

Im Gegensatz zum deutschen Vermögensrecht, vgl. § 4 Abs. 2, 3 VermG. Zu den (wenigen) Ausschlussgründen im südafrikanischen Recht J. von Bismarck, (Fn. 55), 103 ff.

Selbst bei mehrfachen Ansprüchen hinsichtlich derselben Liegenschaft gilt nicht wie im deutschen Vermögensrecht zwingend das Prioritätsprinzip, wonach sich der zeitlich zuerst Enteignete/Vertriebene durchsetzt ( $\$ 3$ Abs. 2 VermG).

Deren Zusammensetzung, Kompetenzen und Verfahren regeln präzise die Art. 4 ff. RLRA. Sie ist verwaltungsorganisatorisch dem Department of Land Affairs untergeordnet.

Zum Staatsaufbau vgl. Kapitel 3 VerfRSA (,Co-operative Government“). Siehe auch I. Currie/ J. de Waal (Hrsg.), (Fn. 16), 119 ff.

72

Informationen zum Gericht mit Sitz in Randburg bei http://wwwserver.law.wits.ac.za/lcc/about. html. Siehe auch Art. 22 ff. RLRA.

73

Gemäß der Rechtsgrundlage Art. 42C RLRA können der Landminister oder ein Delegat derartige Hilfen auch nach erfolgreichem Abschluss von Restitutionsfällen gewähren. 
Art. 38A ff. RLRA ist auch eine Direktanrufung des Courts und somit eine eigene Ermittlungstätigkeit des Antragstellers möglich.

Verurteilt der Court am Ende zur Rückübertragung von Grundstücken, ${ }^{75}$ ist diese ggf. im Wege der Enteignung des derzeitigen Eigentümers unter Hinzuziehung der Regeln des vorkonstitutionellen, allgemeinen Expropriation Acts von 1975 (EA) durch den Landminister durchzuführen. ${ }^{76}$ Der RLRA ist vorgehende lex posterior, soweit Überlagerungen vorhanden sind.

Der Verweis auf den EA in Art. 35 Abs. 5A RLRA, der bis zum Jahre 2004 bestand, enthielt teilweise Überlagerungen des alten Enteignungsrechts (bei dessen Erlass an sich vor allem an Verkehrswegeplanung und Grundstücke für öffentliche Einrichtungen gedacht wurde) ${ }^{77}$ für den neueren Sonderfall der Landrestitution. Ermächtigungsgrundlage für den Eingriff im EA war Art. 2. Im Einzelnen geregelt sind im EA insbesondere ein rechtsstaatliches Notifizierungsverfahren (Art. 7, 9) und das Zustandekommen und die Bemessung der Entschädigung (Art. 10 ff.). Anstelle der 2004 abgeschafften Verweisungsnorm des Art. 35 Abs. 5A RLRA wurde mit Art. 42E eine direkte Grundlage zur Enteignungsermächtigung für den Landminister eingeführt, die - und das ist eine bemerkenswerte Änderung - auch für Fälle gilt, in denen mangels Antragstellung zwar kein Anspruch nach dem RLRA gerichtlich festgestellt wird, das zu enteignende Grundstück aber in Beziehung ${ }^{78} \mathrm{zu}$ einem möglichen Anspruch steht. Für die Entschädigung wird immer noch neben dem EA 1975 jetzt unmittelbar Art. 25 VerfRSA in Bezug genommen. ${ }^{79}$ Die wie ein scharfes Schwert anmutende Regelung hat ihre Daseinberechtigung aufgrund von Art. 25 Abs. 5 VerfRSA, der insbesondere den Gesetzgeber zu ebensolchen proaktiven Maßnahmen zur Landverschaffung für jedermann anhält.

J. von Bismarck, (Fn. 55), 108 ff., 139 f., der den Grundsatz der Rückübertragung als umfassende Befriedigung einleuchtend anhand einer Auslegung nachweist.

Art. 35 Abs. 1 a) RLRA.

Der Expropriation Act eröffnet in Art. 26 die Möglichkeit zu sondergesetzlichen Enteignungsrechtsgrundlagen, bestimmt aber, dass die in ihm festgelegten Grundsätze über die Entschädigung unberührt bleiben.

77 Aus diesem Grunde ist prinzipiell auch der Minister of Public Works (Bauminister) für Enteignungen zuständig.

Im neuen Art. 42E RLRA heißt es: ,related to or affected by such claim“.

Kritikwürdig an der fortbestehenden Bezugnahme auf den EA mag erscheinen, dass es in Art. 2 EA schlicht heißt, die Enteignung sei nur gegen Entschädigung zulässig (,subject to an obligation to pay compensation“), und mithin noch keine Anpassung an die diesbezüglichen Wertungen der jüngeren VerfRSA zur Landfrage stattgefunden hat, und ebenso dass bei Berechnung der Entschädigungshöhe nach dem EA lediglich der Marktwert als Obergrenze festgelegt wird. Die Adjektive "just and equitable" und die Maßstäbe des Art. 25 VerfRSA sind aber wegen des Vorrangs der Verfassung) in die Rechtsgrundlagen hineinzulesen. 
Kraft Überlagerung des EA durch den RLRA gelten auch die Regelungen über das Zustandekommen der Entschädigungshöhe im Restitutionsfall zum Zwecke der Verfahrenskonzentration nur eingeschränkt. ${ }^{80}$ Schon der Land Claims Court kann die Entschädigungshöhe nach den verfassungsrechtlichen Wertungen festsetzen, womit das Gesetz den Verfassungsvorgaben für den Fall der fehlenden Einigung (Art. 25 Abs. 2 VerfRSA, siehe oben) gerecht wird. ${ }^{81}$ Ein Rechtmittel gegen die Endentscheidung ist nur zum Supreme Court of Appeal oder zum Constitutional Court zulässig.

\section{Vergleichender Exkurs zum deutschen Enteignungs- und Vermögensrecht}

Zur Veranschaulichung dieses Regelwerks soll ein kurzer Blick auf das deutsche Enteignungsrecht und die hiesige Problematik der offenen Vermögensfragen dienen. Je nach Gesetzgebungskompetenz für die jeweilige Sachmaterie nach Art. 70 ff. GG, in der sondergesetzliche Enteignungstatbestände zu Art. 14 Abs. 3 GG politisch gewollt sind, haben in Deutschland Bundes- und Landesgesetzgeber manche einfachen Enteignungsgesetze beschlossen. ${ }^{82}$ Das dazugehörige Verwaltungsverfahren regeln die für die Ausführung von Gesetzen im Grundsatz zuständigen Bundesländer selbst, Art. 83, 84 GG. ${ }^{83}$

In Deutschland besteht kein Bedürfnis zu einer generellen Landreform wie in Südafrika. Im Zusammenhang dieses Aufsatzes aber besonders hervorzuheben ist, dass auch deutsches Restitutionsrecht existiert, namentlich der nach der Wiedervereinigung geschaffene Komplex des Rechts der offenen Vermögensfragen, der die Wiedergutmachung von vermögensrechtlichem DDR-Unrecht zum Gegenstand hat und der auch auf Unrecht während der NSZeit entsprechend ${ }^{84}$ anwendbar ist. ${ }^{85}$ Dort werden ebenfalls rechtsstaatswidrige bzw. vor-

Vorgesehen sind bestimmte Zuschläge, Verzugszinsen und Berücksichtigungsverbote (etwa für wertsteigernde Verwendungen nach Notifizierung), vgl. Art. 12 EA.

Kompetenz gemäß Art. 22 Abs. 1 b) RLRA. Das ansonsten im EA vorgesehene konsensual konstruierte Zustandekommen gilt allerdings im Restitutionsfall nicht: Das Ministerium macht ein Angebot, das der Enteignete, der im Vorfeld die Obliegenheit hat, über die wertbildenden Faktoren und etwaige Verwendungen auf das Grundstück aus seiner Sicht Auskunft zu erteilen, annehmen kann. Bei Nichtannahme wird die Annahme nach Ablauf einer angemessenen Frist fingiert, wenn vorher nicht eine gerichtliche Festsetzung der Höhe beantragt wird.

Bsp. für den Bund: Verkehrswege- und Bauleitplanung, §§ 19 FStrG, 22 AEG, 85 ff. BauGB; für ein Land: § 17 DenkmalSchG Bln.

Bsp.: Landesenteignungsgesetz Berlin vom 14.7.1964 (GVBl. 737), neugefasst am 30.11.1984 (GVB1. 1664), als lex specialis zum VwVfG Bln vom 8.12.1976 (GVBl. 2735, 2898), zuletzt geändert am 24.6.2004 (GVB1. 253).

$\S 1$ VI VermG. 
rechtsstaatliche Eigentumsverletzungen zu korrigieren versucht und ganz ähnliche Grundentscheidungen getroffen und rechtliche Möglichkeiten eröffnet wie im RLRA. ${ }^{86}$ Die Bundesrepublik ist als Rechtsnachfolgerin und Sozialstaat zur Wiedergutmachung gehalten. ${ }^{87}$ Die Grundlagen für die Voraussetzungen von Wiedergutmachung im (Bundes-)Vermögensgesetz $^{88}$ wurden bereits im Einigungsvertrag ${ }^{89}$ (Art. 41 und „Gemeinsame Erklärung“ in Anlage III) gelegt und lassen sich wie folgt zusammenfassen: Es gilt auch hier der umstrittene Grundsatz der „Rückgabe vor Entschädigung“ in gebundener Rechtsfolge (also ohne behördliches Ermessen $)^{90}$, allerdings für einen numerus clausus von im einzelnen bestimmten Tatbeständen. ${ }^{91}$ Der Grundsatz wird durch manche Ausnahmetatbestände Vorrang von Investitionen, Unmöglichkeit der Rückgabe durch städtebauliche Gründe, ${ }^{92}$ redlicher Zwischenerwerb, Verzicht - durchbrochen. ${ }^{93}$ Jene sekundäre Entschädigung ist nach Maßgabe komplexer Sondergesetze zu ermitteln. ${ }^{94}$ In wenigen bestimmten Fällen ist auch eine Übereignung von Ersatzland vorgesehen. ${ }^{95}$ Nach $\S 30$ VermG gehen (wie auch in Südafrika) einvernehmliche Lösungen zwischen den Beteiligten vor, und erst wenn es eine solche nicht gibt, entscheidet die Behörde ${ }^{96}$. Gemäß § 30a VermG lief die Frist für Rückübertragungsansprüche am 31.12.1992 ab. Justitiabel sind Restitutionsstreitigkeiten vor der

Restitution findet allein bei besatzungsbedingten Enteignungen nicht statt, Art. 143 Abs. 3 GG (dazu M. Kirn in: vMünch/Kunig, Grundgesetzkommentar Bd. 3, 5. Aufl. 2003, Rn. 14ff. zu Art. 143) und $\S 1$ VIII VermG. Dazu jüngst BVerfG, NJW 2005, 560.

Einen Vergleich der beiden Restitutionsmodelle bietet J. von Bismarck, (Fn. 55), 254 ff., und zum Vergleich der politischen Ausgangslagen 220 ff.; K. Koppe, (Fn. 13), 195 ff.

87
J. von Bismarck, (Fn. 55), 214 ff., 230.

Neufassung vom 9.2.2005 (BGB1. I 205), zuletzt geändert am 22.9.2005 (BGB1. I 2809). Zur Vertiefung F. J. Säcker, Vermögensrecht, Kommentar zu §§ 1 bis 21 VermG, 1995.

Gesetz vom 23.9.1990, BGB1. II, 885 .

Daher ist die Restitution in Deutschland legislativ geprägt, während in Südafrika weite Spielräume durch Exekutive und Judikative auszufüllen sind.

Entschädigungslose Enteignungen; Enteignungen gegen geringere als DDR-übliche Entschädigungen; staatliche Verwaltung/Volkseigentum; ökonomischer Zwang; unlautere Machenschaften; Vermögen von NS-Opfern im Beitrittsgebiet; rechtsstaatswidrige straf- oder verwaltungsrechtliche Entscheidungen in der DDR. Dazu instruktiv http://www.barov.bund.de und M. Stargardt, ZIP 1990, 1636. In der gesetzlichen Enumerierung liegt eine Absage an eine Totalrevision jeder einzelnen Entscheidung in der DDR, vgl. auch den Grundsatz der Gültigkeit von DDR-Verwaltungsakten in Art. 19 Einigungsvertrag.

Widmung zu Gemeingebrauch, Einbindung in komplexen Siedlungs- und Wohnungsbau, gewerbliche Nutzung oder Unternehmenseinheit.

J. von Bismarck, (Fn. 55), 209 ff.

Enthalten im Entschädigungs- und Ausgleichsleistungsgesetz (EALG vom 27.8.1994, BGB1. I 2624, zuletzt geändert 22.9.2005 BGB1. I 2809).

Nach Maßgabe des $§ 21$ VermG; siehe auch $§ 1$ Abs. 3 LEG Berlin.

Die Aufgaben werden im Wesentlichen durch Landesbehörden ausgeführt; das Bundesamt zur Regelung offener Vermögensfragen hat nur geringfügige Zuständigkeiten, vgl. $§ \S 22$ ff. VermG. 
allgemeinen Verwaltungsgerichtsbarkeit ( $\$ 40$ Abs. 1 VwGO). ${ }^{97}$ Die Angreifbarkeit ist auf Revision zum Bundesverwaltungsgericht beschränkt. ${ }^{98}$

Vom südafrikanischen Restitutionsverfahren unterscheidet sich das deutsche Verfahren insgesamt gesehen vor allem durch seine starke legislative Vorprägung, die zu gebundenen Ansprüchen führt, sowie durch festgelegte Ausschlusstatbestände und durch die nur geringe Einflussnahme der höchsten staatlichen Ebene.

\section{Rechtliche Bewertung des Falles Visser und Fazit}

Wendet man das südafrikanische Recht auf den (wenngleich noch unabgeschlossenen) Fall Visser an, so kann man kurz gesagt keine Rechtsbrüche des südafrikanischen Staates feststellen. Problematisch ist offensichtlich, dass die Umstände des Landerwerbs streitig sind und damit bereits die Frage, auf welcher Schiene der Landreform er zu verorten ist. Zur endgültigen Klärung auch davon dient das gründlich geregelte zweistufige Ermittlungsverfahren vor Land Claims Commission und Land Claims Court.

\section{Bei Bestehen eines Restitutionsanspruchs}

Besteht ein Anspruch der Erbengemeinschaft auf Restitution gemäß Art. 2 RLRA, so kann durch letzteren nach Abwägung aller Umstände ohne weiteres ein Gerichtsbeschluss zur Enteignung ergehen, die der Landminister in einem anschließenden Verfahren gegen Entschädigung ausspricht. Für den grundrechtsrelevanten Enteignungsbeschluss besteht im oben aufgezeigten Zusammenspiel von RLRA und EA eine allgemeine, parlamentarischgesetzliche Grundlage. Das laut Tatbestand öffentliche Interesse liegt auf der Hand, weil die Restitution als öffentliches Interesse schon in der VerfRSA angelegt ist. ${ }^{99}$ Die Rechtsgrundlage sieht auch eine Entschädigung vor, deren Höhe sich nach dem (zur Zeit noch nicht abschließend einschätzbaren) Ergebnis der behördlichen und gerichtlichen Sachverhaltsaufklärung richtet und für die Art. 25 Abs. 3 VerfRSA leitend ist. Der Marktwert, der dort als einer von mehreren zu berücksichtigenden Faktoren genannt ist, wurde unabhängig gutachtlich bestimmt. Der Enteignung steht eine Gutgläubigkeit des Adressaten hinsichtlich

97

98

99

Lediglich Streitigkeiten über die Entschädigungshöhe nach Enteignungen gehören vor die ordentliche Gerichtsbarkeit (Art. 14 Abs. 3 S. 4 GG).

Der verkürzte Rechtsschutz gegen Restitutionsentscheidungen dient in beiden Staaten der beschleunigten Erlangung von Rechtssicherheit. Deswegen ist auch bereits das Widerspruchsverfahren eingeschränkt. Vgl. $\S \S 36,37$ VermG.

Art. 25 Abs. 7 VerfRSA. Dies gilt unabhängig davon, dass die konkrete Rückübertragung nur an einen Privaten erfolgt. Zum Problem der (in Deutschland nicht a priori ausgeschlossenen) Enteignung zugunsten Privater vgl. OVG Hamburg NJW 2005, 105 mit Anmerkung von W. Erbguth, Das Dilemma der Enteignung zugunsten privatnütziger Vorhaben, NordÖR 2005, 55. 
der diskriminierenden Umstände in der Geschichte nicht entgegen. Der Diskriminierte soll sein Eigentum dennoch zurückbekommen können. Die Redlichkeit von Visser ist aber gleichwohl ein „Umstand“ im Sinne Art. 25 Abs. 3 VerfRSA, der in die Abwägung zur Entschädigungsbemessung Einzug halten muss, da sie die Historie seines Eigentumserwerbs betrifft. Bei Redlichkeit wird also der Marktpreis nicht gemindert. ${ }^{100}$

Besonderes Augenmerk ist stets auf die Ausübung des Ermessens zu legen, da hier de jure ein weiter Spielraum zum Finden eines im Einzelfall gerechten Ergebnisses besteht. Gesichert ist vorliegend allen Anscheins, dass das Enteignungsverfahren als am schwersten wiegender Eingriff in das Eigentum hier wegen der Dauer der erfolglosen Einigungsversuche über den Preis die vielfach beschworene ultima ratio des Vorgehens darstellt. Sie ist erforderlich i. S. d. Verhältnismäßigkeitsgrundsatzes geworden. ${ }^{101}$ Die gebotene Entschädigungshöhe erscheint nach unabhängiger Begutachtung eines Sachverständigen verhältnismäßig. Sofern nicht alle Umstände im Sinne des Art. 25 Abs. 3 VerfRSA neben dem Marktwert hinreichend berücksichtigt wurden, bietet immer noch die nachgeschaltete Überprüfung durch den Land Claims Court eine Korrekturmöglichkeit, der, wie von Art. 14 RLRA vorgesehen, bei fehlender Einigung eine ihrerseits revisible Entscheidung zum Restitutionsanspruch treffen wird.

\section{Bei Nichtbestehen eines Restitutionsanspruchs}

Wird der gesetzliche Anspruch nach dem RLRA nicht dargetan, so musste nach der bisherigen Politik Südafrikas (,willing seller / willing buyer“) niemand befürchten, Land gegen seinen Willen zu verlieren. Anderes gilt, wenn zu Enteignungen zwecks Umverteilung, also solchen ohne konkret Restitutionsanspruchsberechtigte, übergegangen wird, wie es jetzt anscheinend dem Willen der Bevölkerungsmehrheit entspricht (siehe oben). Auch dann kann nach dem geltenden Recht, aber unter erhöhten Anforderungen, eine Enteignung zulässig sein.

De lege lata kann diese dann nur gemäß Art. 42E RLRA durch das Landministerium - bei Bezug zu einem (nur) möglichen Anspruch nach dem RLRA, für den aber kein Antrag

Soweit man das Merkmal "Historie des Erwerbs" nicht in einem generellen, d. h. auf die gesamte Gesellschaft bezogenen Sinne versteht, vgl. J. Zimmerman, (Fn. 42), 408 f. mw.N. Dies hätte den Vorteil, dass die Geschichte nicht in langwierigen Verfahren erforscht werden muss. Für ein individuelles Verständnis spricht aber neben dem Argument der Einzelfallgerechtigkeit das bereits gesetzlich vorgesehene Ermittlungsverfahren hinsichtlich der einzelnen Ansprüche.

Art. 36 Abs. 1 e) VerfRSA: „Less restrictive means to achieve the purpose“ sind nicht ersichtlich. 
gestellt wurde $-{ }^{102}$ oder ansonsten gemäß dem nicht hierfür maßgeschneiderten, aber verfassungskonform auszulegenden EA als solchem, also bisher durch das Bauministerium, erfolgen.

Während ersterer Fall hier wohl ausscheiden muss, weil gerade bereits ein Einstieg in die Antragsprüfung erfolgt ist, kommt bei tatsächlichem Nichtbestehen eines Restitutionsanspruchs nur der zweite Fall als rechtliche Grundlage in Betracht. Für die Rechtmäßigkeit einer Enteignung danach ist folgendes zu beachten: Dass auch die reine Umverteilung Staatsziel ist, also an ihr ein öffentliches Interesse besteht, geht wie gezeigt unbestreitbar aus dem Regelbeispiel des Art. 25 Abs. 4 a) VerfRSA hervor. Bei der Prüfung der Verhältnismäßigkeit des Eingriffs nach Art. 36 VerfRSA, die dem Adressaten immer Schutz bietet, stellt sich jedoch in jedem Einzelfall die Frage, wie den Interessen der noch Landlosen möglichst weitgehend nachgekommen werden kann bei möglichst schonender Inanspruchnahme des Enteignungs-Adressaten. Um Willkür zu vermeiden, muss in jedem Falle gefragt werden: Ist eine Totalenteignung erforderlich und angemessen? Welche Teile des Grundstücks werden übertragen? Welche Großfarmen sollen primär herangezogen werden? Wenn es um Umverteilung geht, spielt hier namentlich der allgemeine Gleichheitssatz eine wesentliche Rolle. ${ }^{103}$ Wer qualifiziert sich warum für den Landerwerb? Diese Fragen suggerieren, dass der Staat, wenn er sich an die verfassungsmäßige Ordnung halten will, mit Enteignungen zum Zwecke der Umverteilung äußerst behutsam vorgehen muss. Das Argument bloß langer erfolgloser Verhandlungen und ein nicht nachgewiesener Restitutionsanspruch können allein keinesfalls eine verhältnismäßige Enteignung außerhalb des RLRA tragen. Bei Nichtbestehen eines Restitutionsanspruchs der Erbengemeinschaft nach Molamu käme es also auf wesentlich mehr Umstände an als bisher bekannt sind, um das Urteil der Verfassungsmäßigkeit fällen zu können.

\section{Fazit}

Bislang ist in keiner Weise erkennbar, dass im Fall Visser (ebenso wenig übrigens in den Parallelfällen) Unrecht getan worden wäre. Soweit Art. 42E RLRA jedoch als Grundlage herangezogen wird, besteht eine besondere Anfälligkeit für willkürliche Entscheidungen. Als Fazit kann im Anschluss der Betrachtung der Rechtslage zum Visser-Fall dennoch Folgendes festgehalten werden:

102 Vorliegend kann der Anspruch bestehen, was von der Auswertung der Dokumente abhängt; ein 103 Antrag auf Restitution wurde aber gestellt.

Art. 9 VerfRSA. Ungleichbehandlungen bedürfen einer sachlichen Rechtfertigung, vgl. I. $M$. Rautenbach/E. F. J. Malherbe, (Fn. 46), 321: Nur „,unfair discrimination“ ist verboten. 
Erstens ist zu unterstreichen, dass die südafrikanische Landpolitik als Teil des Zielgefüges der VerfRSA, wenngleich für die betroffenen Farmer schmerzhaft, in einem detailliert geregelten, strikt legalen (also nicht willkürlichen, sondern der rule of law unterlegenen) Rahmen stattfindet. Es wurden bislang keine Hinweise auf ein Ausbrechen aus diesen Rahmen offenbar. Zweitens bedeutet die in Südafrika praktizierte Landreform einerseits eine klare Absage an die Aufrechterhaltung eines ungerechten status quo und andererseits eine klare Absage an sozialistische Extrempositionen wie die der SACP (s. oben) oder gar der Verstaatlichung allen Landes. Drittens erfolgen die Enteignungen in Südafrika im Gegensatz zu Simbabwe bislang nicht zur allgemeinen Umverteilung von Land, sondern nur zur konkreten Restitution nach Apartheidsunrecht im Einzelfall - oder zumindest mit einem Bezug dazu - im Verfahren nach dem RLRA, d. h. in wesentlich geringerem Umfang, auch wenn die radikale Politik von Mugabe bei Teilen der Bevölkerung populär sein mag. Experten halten es für unwahrscheinlich, dass nunmehr eine massive Enteignungswelle einsetzt. ${ }^{104}$

\section{E. Lösungsansätze für die Landfrage und Ausblick}

Zur Zeit ist das Department of Land Affairs mit der Ausarbeitung eines Plans zur weiteren Umsetzung der Landreform und zu Lösungsansätzen für den Interessenkonflikt befasst. Das ist notwendig, denn soweit das Prinzip ,willing seller / willing buyer“ weiter angewandt wird und Großfarmer tatsächlich exorbitant hohe Preise verlangen, könnte das hehre Anliegen der Armutsbekämpfung und der gerechten Landverteilung tatsächlich zunichte gemacht werden. Maßnahmen zur Erreichung der in den verschiedenen Facetten der Landreform bestehenden Staatsziele werden aktuell viele diskutiert. ${ }^{105}$

Um seiner Verfassung zu praktischer Wirksamkeit zu verhelfen, ist es für Südafrika zunächst empfehlenswert, in mehrerlei Hinsicht etwa auch die rechtswirklichen Erfahrungen Namibias mit dem dortigen Enteignungsrégime genau zu beobachten und sich ggf. auch am Vorbild Windhoeks zu orientieren. Die Hauptunterschiede sind: Namibia enteignet Land nicht nur zum Zwecke der Restitution, sondern strikt für den Marktpreis auch zur Umverteilung, und Namibia reguliert den Eigentumserwerb durch Ausländer (Art. 16 Abs. 2 VerfNam). ${ }^{106}$ Empfehlenswert ist unter engen Voraussetzungen in der Tat der Über-

104

105

Siehe D. Robertson, (Fn. 10).

Zur Evaluation der Erfolge und Defizite des Maßnahmenbündels Bodenreform bis zum Jahr 1999 durch das DLA zusammenfassend aus wirtschaftswissenschaftlicher Sicht und vorsichtig opti106 mistisch N. Göler von Ravensburg, (Fn. 9), 45 f.

Eine solche Einschränkung des freien Grundstücksverkehrs wie in Namibia im letztgenannten Sinne brächte mit sich, dass Land für Regierungsaufkäufe preiswerter wäre. Es ist dennoch eine Gratwanderung, weil damit gleichzeitig Investitionen in die südafrikanische Wirtschaft gehemmt 
gang zu moderaten Umverteilungsenteignungen, die wie festgestellt grundsätzlich schon unter der jetzt geltenden, demokratisch geschaffenen Rechtslage erlaubt und angestrebt sind. Es muss dafür aber zwingend gewährleistet sein, dass staatliche Politik dabei gleichförmig, verlässlich und im Vorhinein berechenbar ${ }^{107}$ ist.

Die oben aufgeworfenen Fragen zur Verhältnismäßigkeit müssen strikt geprüft und im Enteignungsbeschluss dem Adressaten gegenüber begründet werden. Eine positive Maßnahme zur Garantie von Verhältnismäßigkeit ist weiterhin ein Programm zur vernünftigen Teilbarkeit von Großfarmen in kleinere Parzellen, aber noch effizient und rentabel zu bewirtschaftende Einheiten. ${ }^{108}$ Durch Teilenteignungen besteht dann die Möglichkeit, im Einzelfall jetzige Eigentümer nicht gänzlich zu vertreiben und eine Konkordanz zu deren Grundrechten herzustellen. Verletzungen der Eigentumsgarantie können in der Unverhältnismäßigkeit der Zweck-Mittel-Relation liegen und Teilenteignungen können als weniger einschneidende Maßnahmen ihren Zweck u. U. schon ebenso gut erfüllen (vgl. Art. 36 Abs. 1 lit. d, e VerfRSA). Befürwortet man eine weitergehende Umverteilung und den Abschied vom ,,willing seller / willing buyer“ kann der Verhältnismäßigkeitsgrundsatz also nicht genug betont werden.

Die Entschädigungsleistungen müssen so bemessen sein, dass keine Animositäten entstehen können. Dabei sollte man im Rahmen der Kriterien des Art. 25 Abs. 3 VerfRSA grundsätzlich den Marktpreis zugrunde legen und etwaige Abweichungen davon unter ein striktes Rechtfertigungserfordernis stellen. Bei der Diskussion der gutachtlichen Bewertungsmechanismen für Entschädigungen ist es abzulehnen, diese nach dem (oft geringeren) Ertragswert statt nach dem Marktwert zu bemessen. Unabdingbar ist in jedem Falle ebenfalls eine klare Offenlegung der Kriterien sowohl für das „öffentliche Interesse“, für das enteignet wird, als auch zur Gewichtung der für die Abwägung zur Entschädigung relevanten „Umstände“ (Art. 25 Abs. 2, 3 VerfRSA), wobei eine einfach-gesetzliche Konkretisierung der Begriffe - am besten durch eine Anpassung des 30 Jahre alten Expropriation Acts $1975-{ }^{109}$ einer (wenn auch sorgfältig kasuistischen) Rechtsprechung und erst recht bloßen Verwaltungsvorschriften vorzuziehen ist. Die in Bezug genommene, aber magere Regelung

werden. Die beste Lösung ist als Mittelweg wohl ein Einzelfallgenehmigungserfordernis von Rechtsgeschäften betreffend Großfarmen ab einer wesentlichen ha-Zahl, erteilbar etwa durch Wirtschafts- und Landministerium im Einvernehmen. Eine entsprechende Regulierung ist für 2006 geplant, vgl. T. Mbeki, (Fn. 4).

Dazu gehören etwa eine langfristige und öffentlich nachvollziehbare Raumplanung und langfristige Ankündigungen für Enteignungen, die für die Farmer Verwendungen und Investitionen auf ihren Liegenschaften planbar machen.

Subdivision of Agricultural Land Act Repeal Act (1998). Weiter zu prüfen sind in diesem Kontext auch steuerrechtliche Änderungen, etwa damit größere Holdings nicht begünstigt werden, vgl. zu 109 gemachten Ansätzen N. Göler von Ravensburg, (Fn. 9), 36 ff.

Oder einen neu zu schaffenden, spezialgesetzlichen „Land Redistribution Act“. 
im EA müsste ansonsten immer umfangreich verfassungskonform interpretiert werden, was bei derart schwerwiegenden Eingriffen in das Eigentum nicht wünschenswert ist.

Enteignungen zur Umverteilung von Land sind zwar in der VerfRSA vorgesehen (siehe oben), jedoch sind dem sachnahen Landministerium mit dem RLRA einfach-gesetzlich lediglich Rechtsgrundlagen im Rahmen der Restitution an die Hand gegeben. Ihm fehlt die Verwaltungserfahrung mit allgemeinen Enteignungen, die nach dem allgemeinen Expropriation Act nur Sache des Departments of Public Works gewesen sind. Daher ist es sinnvoll, bei der Novellierung des Gesetzes neben einer Absteckung der Maßstäbe der Umverteilungsenteignung und die damit zu verbindenden Entschädigungsleistungen auch eine allgemeine Zuständigkeit des Landministeriums für andere als Restitutionsenteignungen zu begründen. Art. 42E RLRA ist nur ein Anfang, da auch hier ein Restitutionsbezug bestehen muss. Wobei das Tatbestandsmerkmal ,related to or affected by such claim“ in kritischer Weise unbestimmt ist und gerade hier die Gerichte zu einer Willkür- und Verhältnismäßigkeitskontrolle mit Augenmaß aufgerufen sind, damit nicht bei jedem im zweistufigen Verfahren nicht feststellbaren Anspruch nach Art. 2 RLRA quasi durch die Hintertür dennoch eine Enteignung folgen kann. Nur unter all diesen Prämissen ist eine Abkehr von der „policy of willing seller / willing buyer“ zu befürworten.

Dieser Lösungsansatz setzt allerdings eine extensivere Bereitstellung von Haushaltsmitteln voraus, die zum einen notwendig sind für staatliche Landkäufe und faire Enteignungsentschädigungen. Dieses wird schon durch die Mbeki-Administration in die Wege geleitet. ${ }^{110}$ Zum anderen muss hinreichend Personal mit guter Ausbildung in der Landverwaltung für eine unbürokratische und rechtsstaatlich bewusste Landbeschaffung eingesetzt werden. Ein anderer, ebenfalls weiter zu verfolgender Weg zur Umverteilung sind die abgestufte staatliche Unterstützung der ärmeren Schichten bei Landkäufen und ein Darlehenssystem zu Anschaffung und Betrieb von Produktionsmitteln und die Subventionierung armer Kommunen zur Beschäftigung professioneller Planer und Berater mit rechtlichem, technischem und finanziellem Know-how. ${ }^{111}$ Daneben sollten für weitere Kreise Ausbildungsprogramme zur Erlangung agrartechnischen Fachwissens durchgeführt und finanziell gefördert werden. Im Gespräch ist auch die freiwillige Bildung von landwirtschaftlichen Kooperationen und Joint-Ventures von alteingesessenen Betrieben mit neuen Teilhabern, um starre Strukturen im Agrarbereich aufzuweichen. ${ }^{112}$

110

111 Zu den Arten der Subventionierung siehe N. Göler von Ravensburg, (Fn. 9), 37 f. und das Grünbuch des Departments of Land Affairs zur Bodenreform, 1996, 51 und 55. Siehe auch den Provision of Certain Land for Settlement Act.

112

The Economist vom 7.7.2005, Johannesburg, mit einem Beispiel aus der Provinz Mpumalanga: Kapital, Ausrüstung und Ausbildung der Alteigentümer gegen das Land der neu Begünstigten. 
Selbstverständlich wird parallel zu diesen umverteilenden Bestrebungen der Abschluss der Restitution wegen Apartheidsunrechts zügig und bedingungslos mit lückenloser gerichtlicher Prüfungskompetenz verfolgt werden. Hiervon sind noch Hunderttausende Hektar Landes betroffen. Laut Einschätzungen des Department of Land Affairs und der Vizepräsidentin Mlambo-Ngcuka soll das verbleibende Viertel der Anträge innerhalb der nächsten drei Jahre bearbeitet sein. ${ }^{113}$ Und auch die Landbesitzrechtsreform ist weiterzuführen, so dass niemand mehr um seine Existenz fürchten muss und die lähmenden Auswirkungen von Rechtsunsicherheit gebannt wird.

Abschließend gilt es, im Blick zu behalten, wie verantwortungsvoll das im Wesentlichen gut brauchbare Recht ${ }^{114}$ zur Landreform in Südafrika von den Akteuren in Verwaltung und Rechtsprechung zukünftig ausgeführt werden wird, damit Land ausgewogener verteilt wird und der Staat insgesamt langfristig sozial befriedet wird und bleibt. Das von der Regierung im RDP gesteckte Ziel der 30\%igen Umverteilung des Agrarlandes, das das verfassungsrechtliche Staatsziel Landreform konkretisiert, sollte mit Hilfe der angesprochenen Mittel rigoros, jedoch nicht um jeden Preis, angestrebt werden. Dies, wenn auch nicht notwendigerweise bis 2014, so doch innerhalb einer für die Menschen im Land noch absehbaren Zeit. Denn langfristig kann es nur schädlich sein für die Gesamtstimmung und das Vertrauen in Verfassungsstrukturen eines Staates, wenn Ziele nur auf geduldigem Papier bestehen und sich für die Bevölkerungsmehrheit großflächig nichts zum Guten verändert. Der Eindruck würde sich durchsetzen, die Existenz einer Grundordnung sei belanglos. Die Kriminalität würde weiter befördert werden. Die jetzige Kontroverse über die Landfrage und den Fall Visser gibt aber nach allem Anlass zu Optimismus, denn sie findet respektvoll weitgehend auf der Grundlage der differenzierten Verfassungsrechtslage statt, die als Produkt des von den Interessengruppen lange ausgehandelten Kompromisses eine gemeinsame Grundlage ist. Es ist als ein Zeichen für die Bewährung der Verfassung Südafrikas anzusehen, dass diese selbst dabei nicht in Frage gestellt wird.

Bestehende Schwierigkeiten beschreibt T. Veser, Das Parlament (Internet), 2005, http://www.dasparlament.de/2005/49-50/Ausland/003.html.

113

Department of Land Affairs, (Fn. 59), 21. Es handelt sich noch um ca. 22.000 zu bearbeitende Anträge.

114 Siehe auch die Untersuchung von A. M. Gross, (Fn. 43), 464, der Art. 25, 26 VerfRSA als ideal für Gerechtigkeit in Transitionsgesellschaften und als ein Vorbild für den Staat Israel sieht, weil Privateigentum und Besitzverhältnisse geschützt werden, willkürliche Vertreibungen ausgeschlossen sind und gleichzeitig ein Bekenntnis zu Umverteilung und gleichem Zugang zu Land verankert ist. 